\title{
Immune response to rabies vaccination in pediatric transplant patients
}

\author{
Cramer CH II, Shieck V, Thomas SE, Kershaw DB, Magee JC, Lopez \\ MJ. Immune response to rabies vaccination in pediatric transplant \\ patients. \\ Pediatr Transplantation 2008: 12: 874-877. (C) 2008 Wiley Periodicals, Inc. \begin{abstract}
as the success of solid organ transplantation has improved. These during camp. They completed the immunoprophylaxis treatment for rabies exposure outlined by the CDC in the 2003 Red Book. Rabies titers were followed for six to 12 months post-immunization. All five OLT patients were on tacrolimus. All three RT patients were on tacrolimus, mycophenolate mofetil, and prednisone. At the time of exposure median age was $10.0 \mathrm{yr}(8.4-17.3)$. None of the subjects developed rabies. A positive rabies titer, indicative of successful immunization, was present by one month in seven subjects and all subjects by six months. Rabies vaccination in pediatric transplant patients is safe and associated with the successful production of antirabies titers.
\end{abstract} \\ Abstract: Children have become engaged in a wider variety of activities \\ activities can result in exposure to infectious agents for which there are \\ no data documenting the efficacy of standard treatment in children on \\ immunosuppressive therapy. This is a retrospective review of five OLT \\ patients and three RT patients who were potentially exposed to rabies
}

\author{
Carl H. Cramer II', Victoria Shieck ${ }^{2}$, \\ Susan E. Thomas ${ }^{3}$, David B. Kershaw ${ }^{3}$ \\ John C. Magee ${ }^{4}$ and M. James Lopez ${ }^{5}$ \\ ${ }^{1}$ Pediatric Nephrology, Department of Pediatrics and \\ Adolescent Medicine, Mayo Clinic, Rochester, MN, \\ ${ }^{2}$ Transplant Center, ${ }^{3}$ Pediatric Nephrology, \\ ${ }^{4}$ Department of Surgery and ${ }^{5}$ Pediatric \\ Gastroenterology, Department of Pediatrics \& \\ Communicable Diseases, University of Michigan, \\ Ann Arbor, MI, USA
Key words: vaccination - rabies - transplant - pediatrics - immunization
Carl H. Cramer II, MD, Pediatric Nephrology,
200 First Street SW, Rochester, MN 55095, USA
Tel.: +1 5072667960
Fax: +1 5072667891
E-mail: cramer.carl@mayo.edu
Accepted for publication 16 January 2008

Human rabies in the USA has decreased over the past decades, with one to six cases reported per year between 1993 and 2002 (1). Contrary to popular belief, non-hematophagous bats were the most commonly reported transmitting species [19 of the 31 reported cases of human rabies (1)]. Rabies presents as acute progressive encephalitis caused by one of the RNA viruses, Lyssavirus. Especially in the case of bats as the vector, the diagnosis of rabies may be hindered by a lack of a clear history of exposure. A bat bite can be painless and the incision is often $<1 \mathrm{~mm}$ in size. Preventing rabies associated mortality is successful only with the immediate institution of prophylactic measures with essentially $100 \%$ mortality if treatment begins after symptoms are present. Currently, only one case has been described of a patient surviving rabies who did

\footnotetext{
Abbreviations: CDC, Centers for Disease Control and Prevention; FSGS, focal segmental glomerulosclerosis; GFR, glomerular filtration rate; OLT, orthotopic liver transplant; RFFIT, rapid fluorescent focus inhibition titer test; RIG, rabies immune globulin; RT, renal transplant.
}

not receive post-exposure prophylactic treatment (2). Post-exposure prophylaxis remains the only proven treatment available for preventing rabies associated mortality $(3,4)$. An estimated 15000 40000 people residing in the USA receive rabies prophylaxis each year (5).

As the success of pediatric solid organ transplantation grows, an increasing number of transplant recipients are engaged in outdoor activities previously limited to non-transplanted individuals, e.g., summer camps and sports. This expansion of outdoor activities can result in exposure to such infections as rabies. For those exposed to rabies, a series of vaccinations is recommended to provide protection. Currently, in solid organ recipients, there are no data that document an adequate immune response to the rabies vaccination in this special population.

Because of the relative rarity of such exposures, healthcare professionals who care for post-transplant patients must utilize these special circumstances to follow the standard specific medical interventions and determine if these interventions are equally efficacious in the transplanted patient. We report eight pediatric transplant (liver or 
kidney) patients potentially exposed to rabies who were treated in accordance with the 2003 Red Book/CDC recommendation for post-exposure vaccination. All patients received the rabies vaccine series and rabies immunoglobulin therapy. We followed the patients' rabies neutralizing antibody titers to determine if these treatments were efficacious under these special circumstances.

\section{Methods}

Patients

This is a retrospective review of five OLT patients and three RT patients who were exposed to rabies during a week long summer transplant camp for transplant recipients. A nonspeciated bat was identified in the cabin of the eight vaccine recipients, of which one of the individuals had a new skin laceration at the base of the occiput that same morning. All campers in the cabin were vaccinated because of the painless and tiny nature of most bat bites. The following information was collected for each patient: the patient's primary indication for transplant, type of transplant, age at transplant, age at first rabies vaccination, time between transplant and first rabies vaccination, any complications of the vaccination series, type of immunosuppressant medications, and any evidence of allograft dysfunction post-immunization. Institutional Board Review approval was obtained for this retrospective study.

\section{Vaccines}

The most current rabies post-exposure immunization protocol was followed as outlined in the 2003 Red Book by the CDC $(6,7)$. All patients received a single dose of RIG of $20 \mathrm{IU} / \mathrm{kg}$ body weight on day 0 . Patients received a series of five doses of $1.0 \mathrm{~mL}$ of rabies vaccine on days $0,3,7,14,28$ (Table 1). The manufacture of the passive and active immunizations was determined by availability of the immunizations at the treating medical facility.

\section{Serological testing}

Rabies virus neutralizing antibody titers were obtained at one, three, six, and 12 months after vaccination. Samples

Table 1. Passive and active immunization provided to subjects

\begin{tabular}{llll}
\hline Subject & $\begin{array}{l}\text { Transplant } \\
\text { type }\end{array}$ & Passive $^{\mathrm{a}}$ & Active $^{\mathrm{b}}$ \\
\hline 1 & OLT & HyperRab & RabAvert \\
2 & OLT & HyperRab & RabAvert \\
3 & OLT & HyperRab & RabAvert \\
4 & OLT & HyperRab & RabAvert \\
5 & OLT & HyperRab & RabAvert \\
6 & LLRT & BayRab & Imovax \\
7 & DRT & BayRab & RabAvert \\
8 & LLRT & BayRab & Imovax \\
\hline
\end{tabular}

OLT, orthotopic liver transplant; LLRT, living related renal transplant; DRT, deceased donor renal transplant.

apassive Immunization: Brand name/manufacturer; Bayrab (Bayer), HyperRab (Talecris Biotherapeutics).

${ }^{\mathrm{b}}$ Active Immunization: Brand name/manufacture: RabAvert (Chiron Behring), Imovax (Aventis Pasteur). were tested using the RFFIT, which is the standard type of serological testing used by the CDC. Different laboratories were used to measure the antibody response which resulted in different reporting techniques. Titers $>0.5 \mathrm{IU} / \mathrm{mL}$ or "Present" are considered indicative of an efficacious response to the immunization series (such a response is considered to indicate adequate protection for the nonimmunosuppressed population).

\section{Statistical analysis}

Descriptive statistics including mean, median, and standard deviation are reported. The small sample size and the heterogeneity of antibody titer measurements limited further statistical comparison.

\section{Results}

The median age at the time of the rabies exposure for the eight recipients was $10.0 \mathrm{yr}$ (range 8.4 $17.3 \mathrm{yr}$ ). Patients were a median of $4.4 \mathrm{yr}$ (range 0.9-16.1 yr) post-transplant. All were male. All five OLT patients were on tacrolimus as the sole immunosuppressant (goal trough $3-5 \mathrm{ng} / \mathrm{mL}$ ). Original diagnoses were biliary atresia in two patients, acute liver failure in two patients, and progressive familial intrahepatic cholestasis in one patient (Table 2). None had recent complications of their transplant allograft. All three RT patients were on tacrolimus (goal trough 4-6 ng/ $\mathrm{mL}$ for patients 6 and 8; goal trough $6-8 \mathrm{ng} / \mathrm{mL}$ for patient 7), mycophenolate mofetil (500$600 \mathrm{mg} / \mathrm{m}^{2} /$ day divided b.i.d.), and prednisone $(0.15 \mathrm{mg} / \mathrm{kg} /$ day, maximum dose $10 \mathrm{mg})$. Original diagnoses in the renal transplant patients were renal cortical necrosis from sepsis, FSGS, and obstructive uropathy from posterior uretheral valves.

None of the subjects developed rabies in the one-yr period following exposure. No patient had any severe adverse reaction to the vaccinations. No patient developed allograft rejection. In the OLT patients, the mean titers (IU/ $\mathrm{mL}) \pm$ s.d. at one, three, six, and 12 months were $8.99 \pm 3.22, \quad 3.78 \pm 2.13,2.90 \pm 1.19$, and $2.20 \pm 1.39$, respectively. The mean titers could not be measured for the remaining patients because the results were reported either qualitatively or above certain thresholds. However, after six months of follow-up all patients demonstrated an efficacious response to the vaccine.

Patient 4 had a liver biopsy one-month postvaccination for chronic elevation of liver enzymes at approximately $1.5 \times$ normal. The biopsy demonstrated chronic hepatitis with mild features suggestive of autoimmune-like changes. His liver tests remained stable during the one-yr follow-up period without any change in management. Patient 7 had post-transplant recurrence of 
Table 2. Characteristics of transplant subjects who received rabies vaccination and their rabies antibody titer response

\begin{tabular}{|c|c|c|c|c|c|c|c|c|}
\hline \multirow[b]{2}{*}{ Subject } & \multirow[b]{2}{*}{ Primary diagnosis } & \multirow{2}{*}{$\begin{array}{l}\text { Type of } \\
\text { ftransplant }\end{array}$} & \multirow{2}{*}{$\begin{array}{l}\text { Age (yr) } \\
\text { at exposure }\end{array}$} & \multirow{2}{*}{$\begin{array}{l}\text { Years } \\
\text { post-transplant }\end{array}$} & \multicolumn{4}{|c|}{ Rabies titer post-immunization (IU/mL) } \\
\hline & & & & & 1 month & 3 months & 6 months & 12 months \\
\hline 1 & Biliary atresia & OLT & 17 & 16.1 & 11.69 & 6.23 & 4.23 & 4.09 \\
\hline 2 & Familial intrahepatic cholestasis & OLT & 9 & 2.6 & 4.91 & 2.8 & 2.54 & 1.59 \\
\hline 3 & Biliary atresia & OLT & 10 & 9.3 & 7.93 & NA & 1.93 & 0.84 \\
\hline 4 & Idiopathic acute liver failure & OLT & 10 & 4.6 & 11.44 & 2.32 & NA & 2.27 \\
\hline 5 & Idiopathic acute liver failure & OLT & 10 & 8.2 & Present & Present & NA & Present \\
\hline 6 & Cortical necrosis & LRRT & 9 & 4.2 & $>1.0$ & Present & $\geq 0.8$ & NA \\
\hline 7 & Focal segmental glomerulosclerosis & DRT & 9 & 0.9 & $\leq 0.2$ & $\leq 0.2$ & $\geq 0.9$ & NA \\
\hline 8 & Obstructive uropathy & LRRT & 9 & 1.1 & $>1.2$ & $\geq 0.8$ & $\geq 0.8$ & NA \\
\hline
\end{tabular}

OLT, orthotopic liver transplant; LLRT, living related renal transplant; DRT, deceased donor renal transplant.

Titers $>0.5 \mathrm{IU} / \mathrm{mL}$ or "present" are considered "protective."

NA, titers are not available.

his focal segmental glomerular sclerosis within the first month of his transplant and predated the rabies exposure. He initially received plasmapheresis, but this was discontinued prior to his potential rabies exposure. He had an estimated GFR of $25-30 \mathrm{~mL} / \mathrm{min} / 1.73 \mathrm{~m}^{2}$ during the study period.

\section{Discussion}

Rabies immunization resulted in antibody titers above the protective cutoff in all but one patient by one month. The results outlined in Table 2 support all subjects responded to the immunization, with positive rabies titers by six months post-vaccination. The administration of human rabies immune globulin alone rarely results in protective rabies titers (8). Therefore, the antibody titers seen at one month in our patients are likely the results of an immune response to the cell culture vaccines. Our heterogeneous study with a limited number of subjects limits the ability to determine if one brand of vaccine is superior in this transplant population. Also, we are not able to determine if there is a difference in antibody titer response between the liver and renal transplant patients as different reporting mechanisms and immunosuppression regiments were used. However, the antibody response appears to be less than a group of 19 immunocompetent children ages $7-10$ who received the rabies vaccine series. Their immune response demonstrated an antibody response range of 3-33 IU/mL (mean $21 \mathrm{IU} / \mathrm{mL}$ ) (9).

In renal transplant patient 7 , protective titers were not seen until six months after vaccination. The impaired titer response to the vaccine in patient 7 may have been secondary to his active FSGS (nephrotic syndrome) and stage IV chronic renal failure. The rise in titers is likely related to clinical improvement in his nephrotic syndrome. One surrogate marker of his nephrotic syndrome that was consistently measured throughout the study was his serum albumin. At the time of his immunization his serum albumin was $2.1 \mathrm{~g} / \mathrm{dL}$, then $2.4 \mathrm{~g} / \mathrm{dL}$ at three months, and finally $3.0 \mathrm{~g} / \mathrm{dL}$ at six months. Patient 7 was not receiving plasmapheresis at the time of his exposure or immunizations.

Rabies vaccination in pediatric transplant patients is safe and efficacious while receiving low to modest amounts of immunosuppression. Specifically, all of the subjects demonstrated a response to the active immunization at six and 12 months. We hope this helps address one of the potential barriers the pediatric transplant patient may encounter when pursuing many of the activities of non-immunosuppressed children. This "natural experiment" provides important information that this patient population should receive the $\mathrm{CDC}$ recommend rabies vaccination schedule if clinically indicated.

\section{Acknowledgment}

This manuscript was presented in abstract form at the 2007 American Society of Transplantation Meeting in San Francisco, CA, USA.

\section{References}

1. Belotto A, Leanes LF, Schneider MC, et al. Overview of rabies in the Americas. Virus Res 2005: 111: 5-12.

2. Willoughby RE, Tieves KS, Hoffman GM, et al. Survival after treatment of rabies with induction of coma. N Engl J Med 2005: 352: 2508-2514.

3. Bahmanyar M, Fayaz A, Nour-Salehi S, et al. Successful protection of humans exposed to rabies infection: Postexposure treatment with the hew human diploid cell rabies vaccine and antirabies serum. JAMA 1976: 236: 2751-2754. 
4. Human Rabies prevention - United States, 1999: Recommendations of the Immunization Practice Advisory Committee (ACIP). MMWR Recomm Rep 1999;48:1-21.

5. Krebs JW, Long-Marin SC, Childs JE. Causes, costs, and estimates of rabies post-exposure prophylaxis treatments in the United States. J Public Health Manag Pract 1998: 4: 56-62.

6. Pickering L, ed. Red Book: Report of the Committee on Infectious Diseases, 26th edn. EIK Grove Village, IL: American Academy of Pediatrics, 2003: pp. 514-521.
7. Rupprecht CE, Gibbons RV. Clinical practice. Prophylaxis against rabies. N Engl J Med 2004: 351: 2626-2635.

8. Lang J, Gravenstein S, Briggs D, et al. Evaluation and safety of immunogenicity of a new, heat-treated human rabies immune globulin using a sham, post-exposure prophylaxis of rabies. Biologicals 1998: 26: 7-15.

9. Willoughby RE, Hammarin AL. Prophylaxis against rabies in children exposed to bats. Pediatr Infect Dis J 2005: 24: 1109 1110 . 